EGU2020-20403

https://doi.org/10.5194/egusphere-egu2020-20403

EGU General Assembly 2020

(c) Author(s) 2020. This work is distributed under

the Creative Commons Attribution 4.0 License.

\title{
A conceptual framework for vulnerability and risk assessment in the context of nature-based solutions to hydro-meteorological risks
}

\author{
Mohammad Aminur Rahman Shah ${ }^{1}$, Fabrice G. Renaud ${ }^{1}$, Annie Wild ${ }^{1}$, Carl C. Anderson ${ }^{1}$, Michael \\ Loupis ${ }^{2,3}$, Depy Panga ${ }^{2}$, Maria Stefanopoulou ${ }^{2}$, Annemarie Polderman ${ }^{4}$, Eija Pouta ${ }^{5}$, Athanasios \\ Votsis $^{6}$, Craig Thomson ${ }^{7}$, Karen Munro ${ }^{7}$, Bidroha Basu ${ }^{8}$, Francesco Pilla ${ }^{8}$, Beatrice Pulvirenti ${ }^{9}$, Elena \\ Toth $^{10}$, Alessio Domeneghetti ${ }^{10}$, and Silvana Di Sabatino ${ }^{11}$ \\ ${ }^{1}$ School of Interdisciplinary Studies, University of Glasgow, Dumfries, United Kingdom \\ (mohammadaminurrahman.shah@glasgow.ac.uk; Fabrice.Renaud@glasgow.ac.uk; anniewild@gmail.com; \\ 2425748A@student.gla.ac.uk) \\ 2Innovative Technologies Centre (KKT-ITC), 11635 Athens, Greece (mloupis@itcnet.gr; Depy.Panga@itcnet.gr; \\ stefanopoulou@live.com) \\ ${ }^{3}$ General Department, National and Kapodistrian University of Athens, 34400 Psachna, Greece (mloupis@itcnet.gr) \\ ${ }^{4}$ Institute for Interdisciplinary Mountain Research, Austrian Academy of Sciences (OEAW), Innsbruck, Austria \\ (Maria.Polderman@oeaw.ac.at) \\ ${ }^{5}$ Natural Resources Institute Finland (Luke), Finland (eija.pouta@luke.fi) \\ ${ }^{6}$ Weather and Climate Change Impacts, Finnish Meteorological Institute (FMI), Helsinki, Finland (Athanasios.Votsis@fmi.fi) \\ ${ }^{7}$ BEAM Research Centre, Glasgow Caledonian University, Glasgow, United Kingdom (Craig.Thomson@gcu.ac.uk; \\ Karen.Munro@gcu.ac.uk) \\ ${ }^{8}$ School of Architecture, Planning and Environmental Policy, University College Dublin, Belfield, Ireland \\ (bidroha.basu@ucd.ie; francesco.pilla@ucd.ie) \\ ${ }^{9}$ Department of Industrial Engineering, University of Bologna, Bologna, Italy (beatrice.pulvirenti@unibo.it) \\ ${ }^{10}$ Department of Civil, Chemical, Environmental and Materials Engineering, University of Bologna, Bologna, Italy \\ (elena.toth@unibo.it; alessio.domeneghetti@unibo.it) \\ ${ }^{11}$ Department of Physics and Astronomy, University of Bologna, Bologna, Italy (silvana.disabatino@unibo.it)
}

Various frameworks for vulnerability and risk assessment of social-ecological systems (SES) to natural hazards have been developed addressing different contexts. However, none were specifically developed in the context of implementing nature-based solutions (NBS) to hydrometeorological risks. Since the basic concepts and principles of NBS are mainly focused on ensuring balance between ecological and social benefits, the entire vulnerability and risk assessment process should focus equally on various social and ecological components of a location where an NBS would be implemented. As a part of the OPEn-air laboRAtories for Nature baseD solUtions to Manage hydro-meteo risks (OPERANDUM) project, this research proposes a conceptual framework for vulnerability and risk assessment in the context of NBS to hydrometeorological risks. This conceptual framework is developed mainly considering the major components of the existing Delta-SES risk assessment framework (Sebesvari et al. 2016) and other similar frameworks proposed in recent studies, as well as the proposed principles for NBS endorsed by International Union for Conservation of Nature (IUCN). The major components of the framework include: (i) the exposure of SES to multiple hydro-meteorological hazards (e.g., flood, 
drought); (ii) vulnerability of SES that consists of ecosystem susceptibility, social susceptibility, ecosystem robustness, and coping and adaptive capacity of the social system; (iii) risks in the NBS project site determined by the combination of hazard exposure and vulnerability; and (iv) the impacts of hydro-meteorological hazards on the SES surrounding or within the NBS project site. While the basic space of risk assessment would be the NBS project site (usually at the local level within sub-catchments) with specific SES characteristics, this framework also reflects the interrelationships between ecosystem and social system as well as the effects of multiple hazards and risks at local up to the global scales. The framework also considers the changes over time that would capture the maturation time lag of the ecological components of an NBS, as well as the sustainability of the system with the intervention of NBS and other risk reduction measures. An indicator-based risk assessment approach can be used to operationalize the framework. To facilitate that, an indicator library has been developed comprising of indicators for different exposure and vulnerability components of the framework. The proposed framework can be applicable to any geographical conditions where an NBS project is to be implemented to reduce hydro-meteorological risks. The framework can also be tailored for other natural hazards (e.g. geological hazards like earthquake) and anthropogenic hazards (e.g. pollution). We will explain the conceptualisation process of the framework and of the indicator library and how these will be tested within the OPERANDUM project in the context of NBS implementation.

Keywords: Nature-based solutions, risk assessment framework, hydro-meteorological hazards, social-ecological systems

\section{Reference:}

Sebesvari, Z., Renaud, F. G., Haas, S., Tessler, Z., Hagenlocher, M., Kloos, J., ... \& Kuenzer, C. (2016). A review of vulnerability indicators for deltaic social-ecological systems. Sustainability Science, 11(4), 575-590. 